\title{
Revisión sobre las características metodológicas y la eficacia de intervenciones orientadas a reducir el consumo de agua*
}

\section{Review about methodological characteristics and efficacy of interventions oriented to reduce water consuming}

\author{
Sergio Vila-Tojo \\ Universidad de Santiago de Compostela, España \\ ORCID: http://orcid.org/0000-0001-8065-9881 \\ Elena Andrade \\ Universidad de Santiago de Compostela, España \\ ORCID: http://orcid.org/0000-0002-0927-7992 \\ Jose Manuel Sabucedo \\ Universidad de Santiago de Compostela, España \\ ORCID: http://orcid.org/0000-0002-3002-851X \\ Sara González-García \\ Universidad de Santiago de Compostela, España \\ ORCID: http://orcid.org/0000-0002-2553-0863 \\ María Teresa Moreira \\ Universidad de Santiago de Compostela, España \\ ORCID: http://orcid.org/0000-0001-9354-3298 \\ Gumersindo Feijoo \\ Universidad de Santiago de Compostela, España \\ ORCID: http://orcid.org/0000-0001-6231-3887
}

a Autor de correspondencia. Correo electrónico: sergiovila.tojo@usc.es

Para citar este artículo: Vila-Tojo, S., Andrade, E., Sabucedo, J. M., González-García, S., Moreira, M. T., \& Feijoo, G. (2019). Revisión sobre las características metodológicas y la eficacia de intervenciones orientadas a reducir el consumo de agua. Universitas Psychologica, 18(5), 1-15. https://doi.org/10.11144/Ja veriana.upsy18-5.rscm

\section{RESUMEN}

Las intervenciones para promover conductas conservadoras del agua han seguido 3 tipos de estrategias: feedback, normas sociales y campañas de sensibilización. Esta revisión pretende evaluar las características metodológicas y valorar la eficacia alcanzada por estas estrategias. De una búsqueda sistemática de literatura, obtuvimos 31 artículos. En cada estrategia, analizamos 6 variables metodológicas esenciales, y calculamos el tamaño del efecto promedio. Encontramos ausencia destacada de grupo control y de medidas pre-post. El tamaño del efecto fue medio en las intervenciones de feedback y bajo en intervenciones basadas en normas sociales y campañas de sensibilización. Las intervenciones de feedback presentan mejores condiciones metodológicas. No obstante, se impone la necesidad de cuidar aspectos metodológicos esenciales, y de informar de estadísticos que permitan una estimación más fiable de la eficacia. Pese a ello, la tendencia de las tres estrategias es positiva, donde las intervenciones basadas en feedback son las de mayor efecto.

Palabras clave

conservación del agua; feedback; normas sociales; campañas de sensibilización; características metodológicas. 


\begin{abstract}
Three types of interventions have been implemented to promote water conservation behaviours: feedback, social norms, and sensitivity campaigns. The aim of this review is to assess methodological aspects and to estimate the efficacy of these interventions. We selected 31 articles through a systematic literature search. For each strategy, we analysed 6 essential methodological variables and we calculated the average effect size. We found a noticeable lack of control group and pre-post measures. The effect size was medium in feedback interventions and small in social norms and sensitivity interventions. Feedback strategies reported better methodological conditions. However, it is necessary to improve these essential methodological aspects and to report statistics which allow a more reliable estimation of efficacy. Despite this, there is a positive tendency of the three strategies, with feedback interventions having a greater effect.
\end{abstract}

Keywords

water conservation; feedback; social norms; sensitivity campaigns; methodological issues.

Los estudios sobre la gestión del agua se han incrementado en los últimos años como respuesta a informes de organizaciones internacionales que alertan sobre la condición actual de las reservas de agua y del proceso de sequía (Organización para la Cooperación y el Desarrollo Económicos, 2016; Naciones Unidas, 2016). Además de las alternativas de reutilización del agua (Hurlimann, Hemphill, McKay, \& Geursen, 2008), una línea de investigación especialmente en aumento desde las perspectivas psicológicas es el desarrollo de estrategias con el objetivo de reducir el consumo del agua (SarabiaSánchez, Rodríguez-Sánchez, \& Hyder, 2014). En concreto, esta perspectiva trataría de evitar el mal uso o el gasto excesivo y superfluo de este recurso natural.

El estudio de los factores determinantes de la conducta de conservación de agua ha derivado en la elaboración de modelos que incidían principalmente en aspectos sociales (Corral-Verdugo, Frías-Armenta, PérezUrias, Orduña-Cabrera, \& Espinoza-Gallego, 2002), o en aspectos fundamentalmente económicos (Renwick \& Archibald, 1998). Con posterioridad, estos paradigmas confluyeron en un modelo integrador propuesto por Jorgensen, Graymore y O'Toole (2009), que incluye factores sociodemográficos, estructurales (como las instalaciones o la composición familiar), económicos (como el tipo de tarifa), climáticos y psicológicos (como las normas).

Identificar estos factores ha permitido la elaboración de intervenciones, que mayoritariamente se encuadran en tres tipos: feedback, normas sociales y campañas de sensibilización. En primer lugar, se estudió la incidencia del feedback sobre el consumo de agua. Ese tipo de intervención pretende modificar el comportamiento de conservación de agua mediante el informe del consumo actual, que en algunas ocasiones contempla valoraciones positivas o negativas sobre dicho consumo. El feedback genera una situación de autoconocimiento, que, al vincularse con mecanismos de condicionamiento operante, actúa como estrategia en la regulación conductual (Skinner, 1974). Mediante la notificación al individuo de su propia conducta, este mantiene o modifica aspectos de la misma y valora las consecuencias de tal acción. La importancia del feedback radica en el desconocimiento del usuario sobre su propio consumo de agua. En este sentido Beal, Stewart, Spinks y Fielding (2011) observaron una sobrestimación del consumo en viviendas pequeñas, con instalaciones poco eficientes, bajos ingresos y un menor número de niños; mientras que los residentes de viviendas grandes, con instalaciones más eficientes, altos ingresos y un mayor número de niños infraestimaron su consumo de agua.

El uso del feedback en este contexto consiste, habitualmente, en el envío por correo de información detallada sobre el consumo (Aitken, McMahon, Wearing, \& Finlayson, 1994). Sin embargo, en los últimos años se ha producido un avance en el diseño de dispositivos a tiempo real, que revelan el potencial que tiene la automatización sobre el cambio de conducta (Arroyo, Bonanni, \& Selker, 2005). El feedback posiciona a los usuarios como agentes activos en el proceso de cambio, aspecto que se ve reforzado a través de la provisión de información inmediata, que permite a los usuarios comprender y controlar el uso de agua (Davies, Doolan, van den Honert, \& Shi, 2014). 
Aquí subyace la importancia de remarcar la preferencia de los usuarios sobre el contenido del feedback. En particular, Liu, Giurco y Mukheibir (2015) encontraron que los indicadores del volumen de agua consumida son considerados de mayor utilidad que indicadores como la duración o la frecuencia de uso. En resumen, al proporcionar feedback se vinculan estrechamente el comportamiento de uso del agua y las consecuencias asociadas. Por ello, y para facilitar a los usuarios la gestión de su consumo, se ha contemplado la posibilidad de desglosar los informes de uso del agua en actividades específicas, relativas, por ejemplo, a la ducha o al grifo (Fielding et al., 2013; Nguyen, Stewart, \& Zhang, 2013). Además, el potencial del feedback no se limita exclusivamente a proveer información, sino que permite integrar elementos simultáneos como la "validación social" (Arroyo et al., 2005). Así, Schultz et al. (2016) evalúan la eficacia de los mensajes normativos a través de la provisión de feedback personalizado sobre el consumo de agua.

Precisamente, las normas sociales constituyen la segunda estrategia abordada en esta revisión. En esencia, son estándares de creencias compartidos entre los miembros de un grupo, y se dividen en dos tipos según la teoría del enfoque de la conducta normativa: lo que la mayoría de la gente hace (norma descriptiva), y el grado de aprobación o desaprobación que los demás tienen sobre un comportamiento determinado (norma prescriptiva) (Cialdini, Reno, \& Kallgren, 1990). El efecto del empleo de normas sociales, tanto descriptivas como prescriptivas, ha sido evaluado no solo sobre conductas directas de reducción del consumo (Schultz et al., 2016), sino también sobre conductas indirectas, como en la reutilización de toallas (Goldstein, Cialdini, \& Griskevicius, 2008). El fomento de normas proambientales, tanto descriptivas como prescriptivas, es una de las estrategias propuestas por autores como Fielding y Hornsey (2016) para afrontar el reto de la sostenibilidad. Sin embargo, también advierten sobre posibles efectos no deseados si los dos tipos de normas sociales no se encuentran alineadas y entran en conflicto. Esto significaría que el comportamiento objeto de estudio sea aprobado, pero no practicado por el grupo, lo que puede distorsionar el efecto deseable.

Finalmente, se ha abordado la introducción de elementos informativos y de concienciación como estrategia de cambio, a la que en posteriores menciones denominaremos "campañas de sensibilización". El conocimiento sobre aspectos vinculados al agua se ha relacionado significativamente con el uso de estrategias cotidianas de ahorro de agua (Dean, Fielding, $\&$ Newton, 2016). De hecho, la propuesta del déficit del conocimiento ha sido ampliamente aceptada como causa de la ausencia de comportamientos sostenibles (Schultz, Khazian, \& Zaleski, 2008). Por este motivo, esta estrategia se ha convertido en la más utilizada para promover comportamientos conservadores (Schultz et al., 2016). Mediante campañas de sensibilización se provee conocimiento sobre la situación actual de los recursos hídricos, y se aportan consejos para mejorar las prácticas de conservación. Sin embargo, en este tipo de intervenciones se suele analizar el consumo poblacional y no el dato pormenorizado de cada vivienda, lo que constituye una limitación en la medida del consumo (Fielding et al., 2013). Además, su eficacia parece estar limitada al corto plazo (Syme, Nancarrow, \& Seligman, 2000). Como mejora en el diseño de este tipo de intervenciones, Trumbo y O'Keefe (2005) plantearon la importancia de fomentar un rol activo en la búsqueda de la información, y no limitarse simplemente a la exposición de esta.

Cada una de las aproximaciones anteriores aporta una estrategia concreta para fomentar el uso racional del agua. Conocer cuál es la más eficaz obliga a establecer, en primer lugar, la fiabilidad y validez de los estudios que emplean estas estrategias, así como la eficacia resultante de las mismas. Por este motivo, el objetivo general de esta revisión es aportar claridad sobre las intervenciones realizadas en la reducción del consumo de agua o el fomento de comportamientos conservadores de agua. Para ello, a) se contrastará la calidad metodológica de los estudios, b) se analizará el grado de eficacia alcanzada por las intervenciones, y c) se aportará 
un resumen cuantitativo de los resultados de los estudios recogidos.

\section{Metodología}

Con el objetivo de reunir el mayor número de intervenciones-experimentos sobre el consumo de agua, se han seguido tres estrategias de búsqueda: a) búsqueda en las bases de datos y meta-buscadores Web of Science, SCOPUS, Google Scholar, PsycINFO, Annual Reviews, Ebsco y Psicodoc; b) búsqueda en las revistas más importantes del área de conocimiento (como Environment and Behavior o Water Resources Research); c) búsqueda manual, a partir de las referencias bibliográficas de los artículos recogidos. La búsqueda fue completada mediante el contacto con los autores, a través de ResearchGate (red social para la comunidad científica).

Como criterios de búsqueda, seleccionamos aquellos artículos posteriores a $1995^{1}$ que aportan datos sobre intervenciones ya aplicadas $y$, específicamente, aquellos que proporcionan una evaluación cuantitativa de su impacto. El criterio temporal abarca más de dos décadas de investigación, que consideramos suficientes para analizar el estado actual de las intervenciones.

Los términos que aparecen a continuación han sido utilizados de forma conjunta para dirigir nuestra búsqueda: "water conservation", "water saving", "water management", "water use", "water consumption", "behaviour", "intention", "concern", "awareness", "knowledge", "change", "modification", “intervention", “experiment”, "motiv*”, "enhance*”, "promot*”, "commitment".

La búsqueda finalizó el 30 de septiembre de 2017. Durante este proceso se registraron en Scopus un total de 9029 resultados, seguidos de Web of Science, con 1482 resultados. Para evitar duplicidades se utilizó el sistema de almacenamiento de archivos Google Drive.

Reunimos un total de 40 artículos para revisión. No obstante, al agrupar las intervenciones por tipo de estrategia, solo tres de ellas (feedback, normas sociales y campañas de sensibilización) conformaban un número razonable de estudios para el posterior análisis. Finalmente, seleccionamos un total 31 artículos que incluyen un conjunto de 35 estudios. La mayor parte son estudios de campo, con diseño quasi-experimetal; aunque también varias investigaciones se han llevado a cabo en condiciones de laboratorio. Debido a la posibilidad de realizar varios tipos de intervención, un mismo trabajo puede estar incluido en varias categorías.

Los aspectos para codificar fueron establecidos por dos investigadores, uno con extensa experiencia metodológica y otro con formación preferentemente en el área de psicología social. Estas características son de naturaleza objetiva, y por tanto no interpretables. Se codificaron los siguientes aspectos metodológicos: la descripción del contexto (entendido como cualquier característica que nos aporte información sobre el contexto en el que se desarrolla la investigación), la descripción de la muestra, la incorporación de grupo control, la inclusión de medidas pre-post, la presencia de procesos aleatorios de selección de muestra o asignación a las condiciones, y el control de variables extrañas.

Las características metodológicas acerca de la existencia de grupo control, medidas prepost, procesos aleatorios y control de variables extrañas fueron codificadas en términos de su presencia o ausencia; mientras que las características sobre la descripción del contexto y descripción de la muestra fueron evaluadas a través de una escala tipo Likert de cuatro niveles: Nada, Poco, Bastante y Mucho. El nivel Nada implica no aportar ningún dato o informar de datos mínimos (nombre del lugar donde se realiza la intervención o el tamaño de la muestra). El segundo nivel, al que hemos denominado Poco, hace referencia a informar de uno o dos datos diferentes a los datos mínimos. El nivel Bastante consiste en informar de tres o cuatro datos. Por último, el nivel Mucho haría referencia a proporcionar más de cuatro elementos en la descripción.

Para valorar con mayor precisión la eficacia resultante de aplicar las distintas técnicas, se calculó el tamaño del efecto usando como 
base el estadístico $d$ de Cohen. A fin de interpretar el tamaño del efecto, recurrimos a los criterios generales propuestos por el mismo autor (Cohen, 1988), que establece como puntos de corte los valores 0.2 (bajo) 0.5 (medio) y 0.8 (alto). Inicialmente este trabajo pretendía ser un metaanálisis. Sin embargo, debido a la información aportada por los estudios, no fue posible la elaboración de un metaanálisis que gozase de unas óptimas condiciones metodológicas. Por este motivo, no ha de confundirse el cálculo del tamaño del efecto de este estudio con la realización de un metaanálisis; se trata simplemente de un informe descriptivo de los resultados cuantitativos aportados por los estudios.

\section{Resultados}

\section{Feedback}

Encontramos un total de 12 estudios que utilizaron la provisión de feedback como herramienta de cambio. Como se puede observar en la Tabla 1, pocos estudios describen significativamente el contexto en el que se realiza la investigación. En concreto, ningún estudio describe Mucho el contexto, mientras que solo el $41.7 \%$ lo describe Bastante. El mismo porcentaje (41.7\%) describe el contexto Poco, y dos de los trabajos no lo describen Nada (16.6\%). Se debe destacar que el $66.7 \%$ informa de la abundancia o escasez de agua de la región. Sin embargo, únicamente el $25 \%$ informa de la existencia de otro tipo de campañas, previas o concurrentes a la intervención del estudio. El análisis del detalle de la muestra presenta resultados similares. Solamente un estudio describe Mucho la muestra $(8.3 \%)$, mientras que el $25 \%$ la describe Bastante. El porcentaje restante se distribuye de forma equitativa $(33.3 \%)$ entre las categorías Poco y Nada.

El $66.7 \%$ de los estudios presentan grupo control, y todos ellos contemplan medidas prepost. Además, aquellos que incluyen algún tipo de proceso al azar sobre la muestra representan el $58.3 \%$. De este porcentaje el
$85.7 \%$ (6/7) incluye procesos de asignación aleatoria a las condiciones, mientras que solo el $28.6 \%(2 / 7)$ afirma seleccionar la muestra al azar. Cabe destacar que Kurz, Donaghue y Walker (2005) consideraron su muestra como pseudoaleatorizada debido a la tasa de respuesta de los participantes. Con relación al control de variables extrañas, el $83.3 \%$ de los estudios informan del control de al menos una variable. El número de residentes por vivienda es la variable que más se controla, presente en un $55.6 \%$ (5/9) del porcentaje previo.

\section{Tabla 1}

Descripción de las características metodológicas en los estudios que utilizan feedback

\begin{tabular}{lcccccc}
\hline \multicolumn{1}{c}{ Autores (año) } & 1 & 2 & 3 & 4 & 5 & 6 \\
\hline Aitken, McMahon, Wearing y Finlayson (1994) & Bastante & Nada & Sí & Sí & Sí & Sí \\
Davies, Doolan, van den Honert y Shi (2014) & Bastante & Bastante & Sí & Sí & No & Sí \\
Fang y Sun (2016) & Poco & Poco & No & Sí & Sí & Sí \\
Fielding et al. (2013) & Bastante & Bastante & Sí & Sí & Sí & Sí \\
Jeong, Gulbinas, Jain y Taylor (2014) & Poco & Poco & Sí & Sí & No & Sí \\
Kappel y Grechenig (2009) & Nada & Nada & No & Sí & No & No \\
Kurz, Donaghue y Walker (2005) & Bastante & Nada & Sí & Sí & Sí & No \\
Liu, Giurco y Mukheibir (2016) & Poco & Mucho & Sí & Sí & Sí & Sí \\
Otaki, Ueda y Sakura (2017) & Poco & Poco & Sí & Sí & Sí & Sí \\
Staats, Harland y Wilke (2004) & Nada & Bastante & Sí & Sí & Sí & Sí \\
Stewart, Willis, Panuwatwanich y Sahin (2013) & Poco & Poco & No & Sí & No & Sí \\
Willis, Stewart, Panuwatwanich, Jones y & Bastante & Nada & No & Sí & No & Sí \\
Kyriakides (2010) & & & & & & \\
\hline
\end{tabular}

Nota. $1=$ iDescribe el contexto?; 2 = iDescribe

la muestra?; 3 = iInforma de la incorporación de grupo control?; 4 = incluye medidas pre y post intervención?; $5=$ iInforma de procesos de selección o asignación al azar?; $6=i$ Informa controlar alguna variable que pudiese influir en los resultados?; Nada $=$ No ofrece datos o datos mínimos (como el nombre del lugar o el tamaño de la muestra); Poco = entre uno y dos datos; Bastante $=$ entre tres y cuatro datos; Mucho = más de cuatro datos.

La amplia mayoría de los estudios (66.7 $\%)$ encuentran resultados positivos en la introducción del feedback como estrategia de reducción del consumo. No obstante, cabe destacar que tres de los cuatro estudios que no hallaron diferencias estadísticamente significativas, e incorporaron tanto medidas pre y post, como grupo control. Con el objetivo de profundizar en si los resultados encontrados reflejan la eficacia del feedback, procedimos a calcular el tamaño del efecto (d) de aquellos estudios que nos aportaron los datos suficientes. Los resultados de siete de estos estudios presentan una gran variabilidad (EffectSize Range $=1.9-0.02=1.88 ; M=0.54, S D$ 
$=0.53,95 \%$ CI $[0.31,0.77])$. Este perfil se mantiene al considerar los cuatro estudios que informan de valores estadísticos pre-post (EffectSize Range $=1.9-0.06=1.84 ; \mathrm{M}=0.94, \mathrm{SD}$ $=0.64,95 \%$ CI $[0.45,1.43])$. Sin embargo, encontramos un menor efecto y una menor variabilidad en los tres estudios que incluían además grupo control (Effect-Size Range $=0.64$ $-0.02=0.62 ; M=0.28, S D=0.22,95 \% \mathrm{CI}$ $[0.15,0.42])$.

\section{Normas sociales}

Los nueve artículos incluidos en este apartado conforman un total de 14 estudios independientes. Es importante destacar que la mitad de los estudios analizaron la reutilización de toallas en hoteles, lo que constituyó una aproximación indirecta a la reducción del consumo. El $71.4 \%$ de los estudios informan de que utilizaron como variable independiente normas descriptivas, y el $42.9 \%$ normas prescriptivas. Conviene destacar que cinco estudios analizaron el efecto de los dos tipos de normas (Morgan \& Chompreeda, 2015; Schultz et al., 2008; Schultz et al., 2016).

En la Tabla 2 se recogen aquellos aspectos metodológicos cuya presencia o ausencia viene determinada por la información que aportan los estudios. La descripción del contexto es escasa. La mayoría de los estudios, un $71.4 \%$, describe Nada o Poco el contexto donde se realiza la intervención, mientras que solo el $28.6 \%$ lo describe Bastante o Mucho. En concreto, un único estudio informa de los períodos de sequía en la región, y seis informan de campañas previas. Algo similar ocurre con la descripción de la muestra. El 64.3 \% no describe Nada la muestra, mientras que el $35.7 \%$ la describe Bastante o Mucho.

Con relación al grupo control, el $78.6 \%$ de los estudios informaron de la existencia de grupo de comparación. Es necesario señalar que en nuestros resultados documentamos aquellos estudios que indicaron la presencia de grupo control. Sin embargo, algunos estudios informaron de un grupo "control" que no cumple dichas condiciones al recibir, por ejemplo, consejos para conservar agua. Es por este motivo por lo que utilizamos el matiz "grupo de comparación". Los estudios que incorporaron una falsa condición control deberían ser entendidos como el cotejo de dos grupos experimentales. Sin embargo, hemos optado por aceptar las consideraciones aportadas por los autores, y no modificar la información original. Por otra parte, el $28.6 \%$ contemplaron medidas pre-post. Cabe destacar que en el estudio de Schultz et al. (2016), si bien es cierto que no se evaluó estrictamente una comparación pre-post, sí se analizó el consumo base, con el fin de comparar a altos y bajos consumidores dentro de las condiciones.

El $71.4 \%$ de los estudios incluyen algún tipo de proceso al azar con relación a la muestra. En concreto, los 10 estudios informan de asignación aleatoria a las condiciones, mientras que solo un único estudio incluye mecanismos de selección aleatoria de la muestra (Ferraro \& Price, 2013). El $64.3 \%$ de los estudios informan controlar al menos una variable, como, por ejemplo, el número de cuartos de baño o el número de dormitorios (Seyranian, Sinatra, \& Polikoff, 2015); o variables más específicas como la cantidad de jabón aplicado al lavar las manos (Richetin, Perugini, Mondini, \& Hurling, 2016). 


\section{Tabla 2}

Descripción de las características metodológicas en los estudios que utilizan normas sociales

\begin{tabular}{|c|c|c|c|c|c|c|}
\hline Autores (año) & 1 & 2 & 3 & 4 & 5 & 6 \\
\hline Ferraro y Price (2013) & Mucho & Nada & Si & Sí & Sí & Sí \\
\hline Fielding et al. (2013) & Bastante & Bastante & $\mathrm{Si}$ & Sí & Sí & Sí \\
\hline Goldstein, Cialdini y Griskevicius (2008) & Nada & Nada & No & No & Sí & No \\
\hline Goldstein et al. (2008b) & Nada & Nada & No & No & Sí & Sí \\
\hline Mair y Bergin-Seers (2010) & Poco & Mucho & No & No & No & Sí \\
\hline Morgan y Chompreeda (2015) & Bastante & Mucho & $\mathrm{Si}$ & No & No & Sí \\
\hline Richetin, Perugini, Mondini y Hurling $(2016)^{\mathrm{a}}$ & Nada & Nada & Si & No & No & No \\
\hline Richetin et al. (2016b) ${ }^{a}$ & Nada & Nada & Si & No & No & No \\
\hline Richetin et al. (2016c) $)^{a}$ & Nada & Nada & Si & Sí & Sí & No \\
\hline Schultz, Khazian y Zaleski (2 & Nada & Nada & $\mathrm{Si}$ & No & Sí & No \\
\hline Schultz et al. $(2008 \mathrm{~b})^{\mathrm{a}}$ & Nada & Nada & $\mathrm{Si}$ & No & Sí & No \\
\hline Schultz et al. $(2008 \mathrm{c})^{\mathrm{a}}$ & Nada & Nada & $\mathrm{Si}$ & No & Sí & No \\
\hline Schultz et al. (2016) & Poco & Mucho & $\mathrm{Si}$ & No & Sí & No \\
\hline Seyranian, Sinatra y Polikoff (2015) & Mucho & Mucho & Sí & Sí & Sí & Sí \\
\hline
\end{tabular}

Nota. $1=$ iDescribe el contexto?; 2 = iDescribe la muestra?; 3 = ¿Informa de la incorporación de grupo control?; 4 = iIncluye medidas pre y post intervención?; 5 = ¿Informa de procesos de selección o asignación al azar?; $6=$ iInforma controlar alguna variable que pudiese influir en los resultados?; Nada $=$ No ofrece datos o datos mínimos (como el nombre del lugar o el tamaño de la muestra); Poco $=$ entre uno y dos datos; Bastante $=$ entre tres y cuatro datos; Mucho = más de cuatro

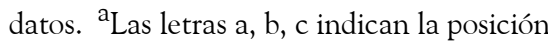
del estudio en un artículo de varios estudios.

Todos los estudios recogidos encontraron una asociación entre la introducción de normas sociales y la reducción del consumo de agua. Morgan y Chompreeda (2015) plantearon diferencias entre los tipos de normas, y encontraron que las prescriptivas fueron las más eficaces, seguidas de la combinación de normas descriptivas y prescriptivas, y de la condición de normas descriptivas. Sin embargo, los resultados de Schultz et al. (2008) indican que presentar las normas de forma combinada es más eficaz que su aplicación en condiciones separadas. $\mathrm{Al}$ calcular el tamaño del efecto de los seis artículos que nos proporcionaban la información esencial, los resultados advierten la existencia de una alta variabilidad (Effect-Size Range $=0.82-0.01=$ $0.81 ; M=0.36, S D=0.27,95 \%$ CI [0.24, $0.47])$. Si solo tenemos en cuenta los valores que contemplan diferencias entre el grupo control y experimental, la magnitud del efecto disminuye (Effect-Size Range $=0.82-0.01=0.81 ; \mathrm{M}=$ $0.34, \mathrm{SD}=0.27,95 \% \mathrm{CI}[0.19,0.49]) . \mathrm{Al}$ analizar los datos sobre las diferencias entre grupo experimental y control en medidas pre-post, el efecto resulta no-significativo (Effect-Size Range
$=0.64-0.02=0.62 ; M=0.23, S D=0.25,95$

$\%$ CI $[-0.08,0.54])$.

\section{Campañas de sensibilización: información y concienciación}

En este apartado se incluyen aquellos artículos que buscan reducir el consumo de agua a través de elementos de concienciación sobre el problema creciente del agua, o a través de elementos informativos, como, por ejemplo, consejos sobre un eficiente uso del agua. Aquí solamente se han tenido en cuenta aquellos estudios que de forma expresa contemplen una condición que incluya estos aspectos. Por tanto, aquellos estudios que incluyen un falso grupo control, en el cual se recibe información, no han sido introducidos en esta categoría.

El número de artículos incorporados después de realizar la búsqueda asciende a 17 , aunque debemos señalar que Lawrence y McManus (2008) analizaron dos programas distintos, y aportaron información pormenorizada para cada uno de ellos, por lo que hemos considerado 18, y no 17 , el total de estudios. Como se observa en la Tabla 3, el $50 \%$ describe Nada o Poco el contexto de la intervención, mientras que el restante $50 \%$ describe el contexto Bastante o Mucho. Cabe destacar que solo el $33.3 \%$ informa de la existencia o ausencia de épocas de sequía, y el $22.2 \%$ informa de la presencia de campañas previas o simultáneas al periodo de intervención. Con respecto a la muestra, el $66.7 \%$ la describe Nada o Poco, mientras que el 33.3 \% la describe Bastante o Mucho.

Del total de 18 estudios, el $55.6 \%$ informó de grupo control, mientras que el $66.7 \%$ de la muestra indicó el registro de medidas pre-post, donde solo el 38.9 \% incluyó ambos aspectos metodológicos. Es importante tener en cuenta las características particulares de dos estudios. Fuss, Bornkessel, Mattern y Stamminger (2011) compararon sus resultados con los obtenidos por otros estudios, por lo que no contemplaron ni grupo control ni medidas pre-post. Por su parte, Watson, Murphy, Kilfoyle y Moore (1999) no diseñaron diferentes condiciones, simplemente 
evaluaron dos fases. En la primera se intervino mediante campañas educativas, y en la segunda se reguló la tarifa del consumo de agua. Aunque los resultados fueron extraídos de dos muestras distintas para cada fase, el resultado de la segunda fase puede estar sesgado por la previa exposición de ambas muestras a la campaña educativa.

El $61.1 \%$ de los estudios incluyeron algún tipo de proceso al azar en relación con la muestra. De este porcentaje, el $54.5 \%(6 / 11)$ realizaron asignación aleatoria a las condiciones y el 63.6 \% (7/11) realizaron selección de la muestra al azar. El 66.7 \% afirman que controlaron alguna variable que pudiese influir en los resultados, como el número de dormitorios o el número de cuartos de baño (Seyranian et al., 2015); o bien variables más específicas, como la temperatura del agua (Fuss et al., 2011) o el formato de los correos enviados (Katz, Grinstein, Kronrod, \& Nisan, 2016).

\section{Tabla 3}

Descripción de las características metodológicas en los estudios sobre campañas de sensibilización

\begin{tabular}{lcccccc}
\hline \multicolumn{1}{c}{ Autores (año) } & 1 & 2 & 3 & 4 & 5 & 6 \\
\hline Ferraro y Price (2013) & Mucho & Nada & Sí & Sí & Sí & Sí \\
Fielding et al. (2013) & Bastante & Bastante & Sí & Sí & Sí & Sí \\
Fuss, Bornkessel, Mattern y Stamminger (2011) & Nada & Nada & No & No & No & Sí \\
Katz, Grinstein, Kronrod y Nisan (2016) & Poco & Nada & Sí & Sí & Sí & Sí \\
Keramitsoglou y Tsagarakis (2011) & Poco & Poco & No & Sí & No & Sí \\
Kurz, Donaghue y Walker (2005) & Bastante & Nada & Sí & Sí & Sí & No \\
Lawrence y McManus (2008)-SS Program $^{\mathrm{a}}$ & Bastante & Nada & Sí & Sí & Sí & Sí \\
Lawrence y McManus (2008)-GH Program $^{\text {b }}$ & Bastante & Nada & Sí & Sí & Sí & Sí \\
Mair y Bergin-Seers (2010) & Poco & Mucho & No & No & No & Sí \\
Middlestadt et al. (2001) & Mucho & Mucho & Sí & No & Sí & No \\
Miller (2010) & Nada & Nada & No & Sí & Sí & No \\
Schultz et al. (2016) & Poco & Mucho & Sí & No & Sí & No \\
Serna (2014) & Mucho & Nada & No & Sí & No & Sí \\
Seyranian, Sinatra y Polikoff (2015) & Mucho & Mucho & No & Sí & Sí & Sí \\
Survis (2016) & Bastante & Nada & Sí & Sí & No & Sí \\
Tijs et al. (2017) & Nada & Poco & No & Sí & Sí & Sí \\
Warner, Chaudhary y Galindo-González (2016) & Nada & Mucho & Sí & No & No & No \\
Watson, Murphy, Kilfoyle y Moore (1999) & Nada & Poco & No & No & Sí & No \\
\hline & & & & & & \\
& & & & & &
\end{tabular}

Nota. $1=i$ Describe el contexto?; $2=i$ Describe la muestra?; 3 = iInforma de la incorporación de grupo control?; 4 = incluye medidas pre y post intervención?; $5=$ ¿Informa de procesos de selección o asignación al azar?; 6 = iInforma controlar alguna variable que pudiese influir en los resultados?; Nada $=$ No ofrece datos o datos mínimos (como el nombre del lugar o el tamaño de la muestra); Poco = entre uno y dos datos; Bastante $=$ entre tres y cuatro datos;

Mucho $=$ más de cuatro datos. ${ }^{\text {aS }}$ Sustainability Street Program, bGreenHome Program

Con respecto a la eficacia, el $83.3 \%$ encontraron una asociación significativa entre la implementación de campañas de sensibilización y la realización de un comportamiento conservador. De forma excepcional, Lawrence y McManus (2008) no obtuvieron diferencias significativas entre los grupos control y experimental en el programa Sustainability Street. También puntualizan que las diferencias encontradas en el programa GreenHome no reflejan una relación causal debido a la tendencia descendente del consumo de agua previa a la aplicación del programa.

Al calcular el tamaño del efecto con los datos de siete estudios, los valores muestran de nuevo una gran variabilidad (Effect-Size Range $=0.65$ - $0.01=0.64 ; M=0.23, S D=0.23,95$ $\%$ CI $[0.08,0.38])$. Obtenemos una amplitud similar al incluir solamente aquellos tamaños del efecto que provienen de las diferencias entre los períodos de pre-post (Effect-Size Range $=0.65$ $0.04=0.61 ; M=0.27, S D=0.25,95 \% \mathrm{CI}$ $[0.04,0.51])$. Si solo tenemos en cuenta aquellos estudios que nos aportan información sobre las diferencias entre el grupo control y experimental, el intervalo de confianza no muestra significación estadística (Effect-Size Range $=0.38-0.01=$ $0.37 ; \mathrm{M}=0.2, \mathrm{SD}=0.19,95 \% \mathrm{CI}[-0.27,0.66])$.

\section{Discusión}

En este artículo presentamos una evaluación de las intervenciones, cuyo objetivo es la reducción del consumo de agua. En general, estas adolecen de los requisitos metodológicos básicos planteados en esta revisión. La descripción aportada del contexto y de la muestra refleja, con escaso detalle, tanto el entorno donde se interviene, como las características de los individuos sobre los que se interviene. Solo un pequeño porcentaje de estudios informa de factores contextuales que pudiesen influir en los resultados de la intervención, como los niveles abundancia del agua. Yazdanpanah, Hayati, Hochrainer-Stigler y Zamani (2014) indican que la medida en que una persona siente miedo respecto a la crisis del agua influye en la intención de conservarla. Por consiguiente, los niveles de abundancia de agua podrían modificar la preocupación o el riesgo percibido de los individuos, influyendo 
así en los resultados de la intervención. De igual forma, la existencia de campañas previas o simultáneas, otro factor poco mencionado, así como los factores estacionales (Otaki, Ueda, \& Sakura, 2017), podrían distorsionar el efecto de la intervención. Un último aspecto también descuidado es el tipo de administración del agua (pública o privada). Investigaciones previas mostraron una relación positiva entre la confianza en las instituciones y aspectos de la percepción de los sistemas de gestión del reciclado de agua (Hurlimann et al., 2008).

Una amplia mayoría de estudios incluye grupo control; no obstante, hemos revisado un número importante de diseños que no contemplan esta característica metodológica, la cual es esencial para la validez de las conclusiones. En particular, la situación es preocupante en la evaluación de las campañas educativas, donde solo el $55.6 \%$ informa de grupo control. Otro indicativo de la validez de los resultados es la presencia de medidas pre-post, la cual varía dependiendo del tipo de estímulo empleado. Todas las intervenciones de feedback incorporan medidas pre y post. Sin embargo, el porcentaje se reduce al $66.7 \%$ en intervenciones educativas, y solo están presentes en el $28.6 \%$ de los estudios de normas sociales. El número de estudios que incluyen mecanismos de asignación o selección aleatoria de la muestra es aceptable, aunque no supera el $75 \%$ en ninguna de las intervenciones. Con relación al control efectivo de variables extrañas, la mayoría de los estudios informan de alguna variable que pudiese distorsionar los resultados. Cabe destacar que los estudios no tienen preferencias sobre el tipo de control realizado, que puede ser metodológico o estadístico. Además, tampoco existe un consenso acerca de aquellas variables sobre las que es necesario ejercer ese control. El modelo integrador sobre la conducta de uso del agua de Jorgensen et al. (2009) puede servir de guía para el control de variables que pudiesen distorsionar el efecto de la intervención. En concreto, destaca la influencia que tienen en el consumo del agua las variables demográficas, las características y composición de la vivienda o los factores climáticos y estacionales.
En una revisión sobre campañas de información, Syme et al. (2000) apuntan a la dificultad de estimar el efecto de las campañas debido a la metodología empleada. Los resultados de nuestra revisión nos permiten concluir en términos similares. Las propiedades metodológicas de las intervenciones sobre el consumo de agua son susceptibles de mejora. Además, si situamos los trabajos en orden cronológico, no observamos una tendencia positiva que indique un avance. Según los aspectos que hemos elegido revisar, en general, podemos concluir que las intervenciones de feedback informan de unas mejores propiedades metodológicas que los restantes tipos de intervenciones.

La eficacia de las intervenciones recogidas en esta revisión es muy variable, y observa tanto efectos grandes como pequeños. Nuestro análisis muestra una reducción de esta variabilidad, y también del tamaño del efecto promedio, al seleccionar únicamente aquellos pocos estudios que incluyen grupo control y medidas pre-post. Esto nos indica la importancia de unas buenas características metodológicas para obtener de forma más precisa el efecto. Es posible que la carencia de mejores propiedades metodológicas derive en la sobreestimación del tamaño del efecto. Con todo y a falta de un metaanálisis, aquellos estudios incluidos en esta revisión presentan un tamaño del efecto promedio medio-bajo. Este efecto también puede verse distorsionado por la tendencia general a realizar diseños transversales. De hecho, tanto la carencia de estudios como la falta de capacidad de las intervenciones para conseguir efectos a largo plazo, son aspectos de preocupación recurrente en este ámbito (Staats, Harland, \& Wilke, 2004).

Además, encontramos una cierta disparidad entre la eficacia resultante aportada por los estudios y el cálculo del tamaño del efecto. Con los datos aportados, y pese a las deficiencias metodológicas y los diferentes métodos empleados, los tres tipos de estrategias obtienen resultados en la misma dirección. Las tres grandes líneas de intervención presentan un impacto positivo en la reducción del consumo 
de agua, aspecto ya recogido en revisiones previas (Inman \& Jeffrey, 2006; Olmstead \& Stavins, 2009). Los resultados sugieren que el feedback conforma una intervención más eficaz en comparación con las normas sociales y las campañas de sensibilización. Sin embargo, no podemos concluir sobre la magnitud de tal efecto, debido a la falta de investigación, o más concretamente, a la falta de provisión de los datos necesarios por parte de los estudios para dicho cálculo.

En este trabajo se puede observar que la mayoría de los estudios realizaron la intervención analizando el consumo de agua total (Otaki et al., 2017), y solamente algunos analizaron comportamientos concretos (Fuss et al., 2011). De estas conductas específicas, solo se han estudiado unas pocas del conjunto de acciones individuales vinculadas a la conservación de agua. También es necesaria mayor indagación sobre el impacto real de las intervenciones. Por ejemplo, es plausible plantear que una intervención de sensibilización aporte no solo conocimiento, sino también responsabilidad ambiental (Maas et al., 2017). Si fuese así, la intervención activaría un elemento motivacional no descrito.

\section{Recomendaciones para futuras intervenciones}

Nuestro trabajo de revisión nos permite señalar una serie de recomendaciones para mejorar el diseño y la información aportada por los estudios dirigidos a reducir el consumo de agua.

La primera recomendación incide en la necesidad de informar de aquellos aspectos relevantes del contexto en el que se realiza la intervención, así como de la muestra utilizada. Aspectos como el clima, los procesos de sequía, los niveles de abastecimiento del agua o la presencia de campañas proambientales son factores imprescindibles para entender el éxito o fracaso de una intervención concreta. Otros más específicos, como la densidad de la población o el número de habitantes del lugar, son aspectos importantes en estudios en los que la unidad de análisis es la vivienda. De igual manera, la descripción de las características sociodemográficas de la muestra, así como su tamaño y los participantes que finalmente son incluidos en el análisis es importante, no solo para entender los resultados de la intervención, sino también para facilitar la ejecución de un metaanálisis de calidad. También sería necesario incluir procesos de selección y asignación aleatoria de la muestra, aunque entendemos que, debido al objeto de la investigación, seleccionar de forma aleatoria la muestra resulta complicado. No obstante, sí sería conveniente un proceso de asignación aleatoria a las condiciones.

En segundo lugar, el diseño de la intervención debería incluir tanto grupo control, como medidas pre-post. El grupo control no debería recibir intervención en ningún momento, ni tampoco ser provisto de consejos o herramientas que faciliten la conservación de agua. En ese caso debería ser considerado como otro grupo experimental dentro de la intervención. Además, se ha de tener especial cuidado en cómo se diseñan las distintas condiciones. Lo ideal sería estudiar cada una de las estrategias de cambio en condiciones separadas, para comprobar el efecto de cada estrategia. En caso de utilizar varias técnicas, se debería considerarlas en conjunto, y concluir en esos términos. La combinación de estrategias, como aportar consejos o información a todas las condiciones experimentales, puede significar que cada condición no refleje el efecto real de la variable de estudio, sino más bien uno acumulado entre la condición y la información.

Otro aspecto para destacar es la necesidad de aportar estadísticos sobre la relación directa entre la intervención y la reducción del consumo de agua. Esto implicaría informar del tamaño del efecto $(d)$ de las diferencias del grupo control y experimental (o de cada uno de los grupos experimentales por separado) en relación con sus medidas pre-post. Si no se aporta el tamaño del efecto, sería necesario aportar valores que permitan calcularlo. Proporcionar esta medida permite que los resultados de la intervención puedan ser incluidos en una revisión cuantitativa, que facilite estimar el impacto de la intervención. 
Finalmente, la investigación debería tener en cuenta el efecto combinado de las tres estrategias. Cada una incide en aspectos diferentes que facilitan el cambio conductual en el individuo. Integrarlas implicaría abordar la problemática de forma holística, que podría revertir en una mayor eficacia en la regulación del uso de agua.

\section{Limitaciones del estudio}

A pesar de haber realizado una búsqueda exhaustiva de artículos, es probable que algún estudio no haya sido incluido debido al amplio número de revistas de diversas disciplinas que publican sobre la gestión del agua. No obstante, consideramos que el conjunto de estudios revisado conforma una base suficiente para comprender el estado actual de las intervenciones sobre la reducción del consumo de agua. Además, es probable la existencia de un sesgo de publicación. La tendencia a publicar resultados significativos frente a los estudios que aportan diferencias no significativas dificulta la interpretación de los resultados.

Es conveniente destacar, además, un posible sesgo cultural en los resultados encontrados. De los 28 estudios que nos aportan información sobre el lugar donde se ha intervenido, el $35.7 \%$ corresponde a poblaciones australianas, y el 32.1 $\%$ a poblaciones estadounidenses.

Por último, una importante limitación es la escasa información aportada por los estudios acerca del tamaño del efecto de la intervención. Esto ha dificultado la realización de un resumen cuantitativo más preciso y fiable.

\section{Agradecimientos}

Esta investigación fue apoyada por el Gobierno de España (AEI), a través del proyecto CTQ2016-75136-P. Los autores pertenecen al Grupo Gallego de Potencial Crecimiento GPC2016-017, y a la Agrupación Estratégica Cretus (AGRUP2015/02). Todos estos proyectos son cofinanciados por Feder (UE).

\section{Referencias}

*Aitken, C. K., McMahon, T. A., Wearing, A. J., \& Finlayson, B. L. (1994). Residential water use: Predicting and reducing consumption. Journal of Applied Social Psychology, 24(2), 136-158. https://www.doi.org/10.1111/j.15 59-1816.1994.tb00562.x

Arroyo, E., Bonanni, L., \& Selker, T. (2005). Waterbot: Exploring feedback and persuasive techniques at the sink. Actas de la SIGCHI Conference on Human Factors in Computing Systems (pp. 631-639) Portland, OG: ACM. https://www.doi.org/10.1145/1 054972.1055059

Beal, C., Stewart, R. A., Spinks, A., \& Fielding, K. (2011). Using smart meters to identify social and technological impacts on residential water consumption. Water Science and Technology: Water Supply, 11 (5), 527-533. https://www.doi.org/10.2166/ws.2 011.088

Cialdini, R. B., Reno, R. R., \& Kallgren, C. A. (1990). A focus theory of normative conduct: Recycling the concept of norms to reduce littering in public places. Journal of Personality and Social Psychology, 58(6), 1015-1026. https://www.doi.org/10.1037/0 022-3514.58.6.1015

Cohen, J. (1988). Statistical power analysis for the behavioural sciences (2⿳亠丷⿵冂丶 ed.). Nueva York, NY: Academic Press.

Corral-Verdugo, V., Frías-Armenta, M., PérezUrias, F., Orduña-Cabrera, V., \& EspinozaGallego, N. (2002). Residential water consumption, motivation for conserving water and the continuing tragedy of the commons. Environmental Management, 30(4), 527-535. https://www.doi.org/10.10 07/s00267-002-2599-5

*Davies, K., Doolan, C., van den Honert, R., \& Shi, R. (2014). Water-saving impacts of Smart Meter technology: An empirical 5 years, whole-of-community study in Sydney, Australia. Water Resources Research, 50(9), 7348-7358. https://www.doi.org/10. 1002/2014WR015812 
Dean, A. J., Fielding, K. S., \& Newton, F. J. (2016). Community knowledge about water: Who has better knowledge and is this associated with water-related behaviors and support for water-related policies? Plos One, 11(7), 1-18. https://www.doi.org/10.1 371/journal.pone.0159063

*Fang, Y. M., \& Sun, M. S. (2016). Applying eco-visualisations of different interface formats to evoke sustainable behaviours towards household water saving. Behaviour $\mathcal{E}$ Information Technology, 35(9), 748-757. https://www.doi.org/10.10 80/0144929X.2016.1189965

*Ferraro, P. J., \& Price, M. K. (2013). Using non-pecuniary strategies to influence behaviour: Evidence from a large scale field experiment. Review of Economics and Statistics, 95(1), 64-73. https://www.doi.org /10.1162/REST_a_00344

Fielding, K. S., \& Hornsey, M. J. (2016). A social identity analysis of climate change and environmental attitudes and behaviors: Insights and opportunities. Frontiers in Psychology, 7, 1-12. https://www.doi.org/10. 3389/fpsyg.2016.00121

*Fielding, K. S., Spinks, A., Russell, S., McCrea, R., Stewart, R., \& Gardner, J. (2013). An experimental test of voluntary strategies to promote urban water demand management. Journal of Environmental Management, 114, 343-351. https://www.do i.org/10.1016/j.jenvman.2012.10.027

*Fuss, N., Bornkessel, S., Mattern, T., \& Stamminger, R. (2011). Are resource savings in manual dishwashing possible? Consumers applying Best Practice Tips. International Journal of Consumer Studies, 35(2), 194-200. https://www.doi.org/10.11 11/j.1470-6431.2010.00972.x

*Goldstein, N. J., Cialdini, R. B., \& Griskevicius, V. (2008). A room with a viewpoint: Using social norms to motivate environmental conservation in hotels. Journal of Consumer Research, 35(3), 472-482. https://www.doi. org $/ 10.1086 / 586910$

Hurlimann, A., Hemphill, E., McKay, J., \& Geursen, G. (2008). Establishing components of community satisfaction with recycled water use through a structural equation model. Journal of Environmental Management, 88(4), 1221-1232. https://ww w.doi.org/10.1016/j.jenvman.2007.06.002

Inman, D., \& Jeffrey, P. (2006). A review of residential demand-side management tool performance and influences on implementation effectiveness. Urban Water Journal, 3(3), 127-143. https://www.doi.org /10.1080/15730620600961288

*Jeong, S. H., Gulbinas, R., Jain, R. K., \& Taylor, J. E. (2014). The impact of combined water and energy consumption eco-feedback on conservation. Energy and Buildings, 80, 114-119. https://www.doi.org/10.1016/j.en build.2014.05.013

Jorgensen, B., Graymore, M., \& O'Toole, K. (2009). Household water use behavior: An integrated model. Journal of Environmental Management, 91(1), 227-236. https://www. doi.org/10.1016/j.jenvman.2009.08.009

*Kappel, K., \& Grechenig, T. (2009). Show-me: Water consumption at a glance to promote water conservation in the shower. Actas del 4th International Conference on Persuasive Technology. Claremont, CA: ACM https://w ww.doi.org/10.1145/1541948.1541984

*Katz, D., Grinstein, A., Kronrod, A., \& Nisan, U. (2016). Evaluating the effectiveness of a water conservation campaign: Combining experimental and field methods. Journal of Environmental Management, 180, 335-343. https://www.do i.org/10.1016/j.jenvman.2016.05.049

*Keramitsoglou, K. M., \& Tsagarakis, K. P. (2011). Raising effective awareness for domestic water saving: Evidence from an environmental educational programme in Greece. Water Policy, 13(6), 828-844. https ://www.doi.org/10.2166/wp.2011.103

*Kurz, T., Donaghue, N., \& Walker, I. (2005). Utillizing a social-ecological framework to promote water and energy conservation: A field experiment. Journal of Applied Social Psychology, 35(6), 1281-1300. https://www.doi.org/10.1111/j. 1559-1816.2005.tb02171.x 
*Lawrence, K., \& McManus, K. (2008). Towards household sustainability in Sydney? Impacts of two sustainable lifestyle workshop programs on water consumption in existing homes. Greographical Research, 46(3), 314-332. https://www.doi.org/10.11 $11 / \mathrm{j} .1745-5871.2008 .00526 . x$

Liu, A., Giurco, D., \& Mukheibir, P. (2015). Motivating metrics for household wateruse feedback. Resources, Conservation and Recycling, 103, 29-46. https://www.doi.org/ 10.1016/j.resconrec.2015.05.008

*Liu, A., Giurco, D., \& Mukheibir, P. (2016). Urban water conservation through customised water and end-use information. Journal of Cleaner Production, 112(4), 3164-3175. https://www.doi.org/10.1016/j.j clepro.2015.10.002

Maas, A., Goemans, C., Manning, D., Kroll, S., Arabi, M., \& Rodríguez-McGoffin, M. (2017). Evaluating the effect of conservation motivations on residential water demand. Journal of Environmental Management, 196, 394-401. https://www.do i.org/10.1016/j.jenvman.2017.03.008

*Mair, J., \& Bergin-Seers, S. (2010). The effect of interventions on the environmental behavior of Australian motel guests. Tourism and Hospitality Research, 10(4), 255-268. https:/ /www.doi.org/10.1057/thr.2010.9

*Middlestadt, S., Grieser, M., Hernández, O., Tubaishat, K., Sanchack, J., Southwell, B., \& Schwartz, R. (2001). Turning minds on and faucets off: Water conservation education in Jordanian schools. The Journal of Environmental Education, 32(2), 37-45. https://www.doi.org/10.1080/00958 960109599136

*Miller, L. L. (2010). Measuring the efficacy of an energy and environmental awareness campaign to effectively reduce water consumption (Disertación doctoral). State College, PA: Pennsylvania State University. Recuperado de https://etda.libraries.psu.edu/catalog/11 337

*Morgan, M., \& Chompreeda, K. (2015). The relative effect of message-based appeals to promote water conservation at a tourist resort in the gulf of Thailand. Environmental Communication, 9(1), 20-36. https://www.d oi.org/10.1080/17524032.2014.917689

Naciones Unidas. (2016). Water and Jobs. París: Unesco. Recuperado de http://unesdoc.une sco.org/images/0024/002439/243938e.pdf

Nguyen, K. A., Stewart, R. A., \& Zhang, H. (2013). An intelligent pattern recognition model to automate the categorisation of residential water end-use events. Environmental Modelling $\mathcal{B}$ Software, 47, 108-127. https://www.doi.org/10.1016/j.en vsoft.2013.05.002

Olmstead, S. M., \& Stavins, R. N. (2009). Comparing price and non-price approaches to urban water conservation. Water Resources Research, 45(4), 1-10. https://ww w.doi.org/10.1029/2008WR007075

Organización para la Cooperación y el Desarrollo Económicos. (2016). Water, growth and finance: Policy perspectives. Recuperado de https://www.oecd.org/environment/reso urces/Water-Growth-and-Finance-policyperspectives.pdf

*Otaki, Y., Ueda, K., \& Sakura, O. (2017). Effects of feedback about community water consumption on residential water conservation. Journal of Cleaner Production, 143, 719-730. https://www.doi.org/10.1016 /j.jclepro.2016.12.051

Renwick, M. E., \& Archibald, S. O. (1998). Demand side management policies for residential water use: Who bears the conservation burden? Land Economics, 74(3), 343-359. https://www.doi.org/10.23 $07 / 3147117$

*Richetin, J., Perugini, M., Mondini, D., \& Hurling, R. (2016). Conserving water while washing hands: the immediate and durable impacts of descriptive norms. Environment and Behavior, 48(2), 343-364. https://www. doi.org/10.1177/0013916514543683

Sarabia-Sánchez, F. J., Rodríguez-Sánchez, C., \& Hyder, A. (2014). The role of personal involvement, credibility and efficacy of conduct in reported water conservation behaviour. Journal of Environmental 
Psychology, 38, 206-216. https://www.doi.or g/10.1016/j.jenvp.2014.02.003

*Schultz, P. W., Khazian, A. M., \& Zaleski, A. C. (2008). Using normative social influence to promote conservation among hotel guests. Social Influence, 3(1), 4-23. https://www.do i.org/10.1080/15534510701755614

*Schultz, P. W., Messina, A., Tronu, G., Limas, E. F., Gupta, R., \& Estrada, M. (2016). Personalized normative feedback and the moderating role of personal norms: A field experiment to reduce residential water consumption. Environment and Behavior, 48(5), 686-710. https://www.doi.org/10.11 77/0013916514553835

*Serna, V. F. (2014). Effects of a water conservation education program on water use in single-family homes in Dallas, Texas. (Disertación Doctoral). Denton, TX: Universidad del Norte de Texas. Recuperado de https://digital.library.unt.ed u/ark:/67531/metadc699967/

*Seyranian, V., Sinatra, G. M., \& Polikoff, M. S. (2015). Comparing communication strategies for reducing residential water consumption. Journal of Environmental Psychology, 41, 81-90. https://www.doi.org/ 10.1016/j.jenvp.2014.11.009

Skinner, B. F. (1974). About Behaviorism. Nueva York, NY: Alfred A. Knopf.

*Staats, H., Harland, P., \& Wilke, H. A. M. (2004). Effecting durable change: a team approach to improve environmental behavior in the household. Environment and Behaviour, 36(3), 341-367. https://www.doi .org/10.1177/0013916503260163

*Stewart, R. A., Willis, R. M., Panuwatwanich, K., \& Sahin, O. (2013). Showering behavioural response to alarming visual display monitors: Longitudinal mixed method study. Behaviour EF Information Technology, 32 (7), 695-711. https://www.do i.org/10.1080/0144929X.2011.577195

*Survis, F. D. (2016). Beyond water restrictions: informing effective lawn watering behavior. (Disertación Doctoral). Boca Ratón, FL: Florida Atlantic University. Recuperado de https://fau.digital.flvc.org/islandora/obje ct/fau\%3A33718

Syme, G. J., Nancarrow, B. E., \& Seligman, C. (2000). The evaluation of information campaigns to promote voluntary household water conservation. Evaluation Review, 24(6), 539-578. https://www.doi.org/10.11 77/0193841X0002400601

*Tijs, M. S., Karremans, J. C., Veling, H., de Lange, M. A., van Meegeren, P., \& Lion, R. (2017). Saving water to save the environment: contrasting the effectiveness of environmental and monetary appeals in a residential water saving intervention. Social Influence, 12(2-3), 69-79. https://www.doi. org/10.1080/15534510.2017.1333967

Trumbo, C. W., \& O'Keefe, G. J. (2005). Intention to conserve water: Environmental values,reasoned action, and information effects across time. Society and Natural Resources, 18(6), 573-585. https://w ww.doi.org/10.1080/08941920590948002

*Warner, L. A., Chaudhary, A. K., \& Galindo-González, S. (2016). Extension participants and nonparticipants differ in water conservation normative beliefs, intentions, and behaviors. Hort Technology, 26(6), 852-861. https://www.doi.org/10.21 273/HORTTECH03484-16

*Watson, R. K., Murphy, M. H., Kilfoyle, F. E., \& Moore, S. M. (1999). An opportunistic field experiment in community water conservation. Population and Environment, 20(6), 545-560. https://www.doi.org/10.10 23/A:1023370100947

*Willis, R. M., Stewart, R. A., Panuwatwanich, K., Jones, S., \& Kyriakides, A. (2010). Alarming visual display monitors affecting shower end use water and energy conservation in Australian residential households. Resources, Conservation and Recycling, 54(12), 1117-1127. https://doi.o $\mathrm{rg} / 10.1016 /$ j.resconrec.2010.03.004

Yazdanpanah, M., Hayati, D., Hochrainer-Stigler, S., \& Zamani, G. H. (2014). Understanding farmers' intention and behavior regarding water conservation in the Middle-East and North Africa: A case study in Iran. 
Journal of Environmental Management, 135, 63-72. https://www.doi.org/10.1016/j.jenv man.2014.01.016

\section{Notas}

[1] Con la excepción de Aitken et al. (1994) por la proximidad a la fecha establecida y su gran relevancia en el campo.

* Artículo de revisión. Indica que los artículos fueron incluidos en la revisión. 